\title{
Application of laser-induced backscattering imaging for predicting and classifying ripening stages of "Berangan" bananas
}

\begin{abstract}
This study investigates the potential of laser light backscattering imaging (LLBI) for predicting and classifying Musa Acuminata cv. Berangan at different ripening stages. Backscattering images of Berangan bananas at various ripening stages from 2 to 7 were acquired by a charge coupled device (CCD) camera coupled with a laser diode emitting light at a wavelength of $658 \mathrm{~nm}$. The grey level intensity and the size of the backscattering area were extracted from the backscattering images and used as parameters for estimating the quality properties of the bananas. The results showed strong correlation between the LLBI parameters and the colour of the bananas as well as the total soluble solids content (TSS) with a coefficient of determination (R2) of over 0.70. Statistical analysis using Linear Discriminant Analysis (LDA) successfully classified the samples into their ripening stages accordingly with the percentage of correct classification up to $94.2 \%$. Thus, it can be concluded that the LLBI could potentially be used for predicting and classifying the ripening stages of bananas and be further developed for an automated quality control system.
\end{abstract}

Keyword: Backscattering imaging; Laser light; Banana ripening; Classification; Imaging 